\title{
Part 12: Internet of Things
}

\author{
Universal Controller: An Open Source Software \\ Development Promoting Connectivity with Assis- \\ tive Technology Devices and the Internet of Things

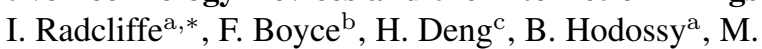

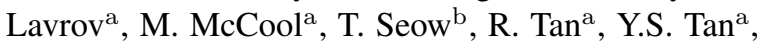 \\ J. Tso ${ }^{\mathrm{d}}$, S.K. Yi ${ }^{\mathrm{a}}$ and B. Mair \\ ${ }^{\mathrm{a}}$ Department of Bioengineering, Imperial College Lon- \\ don, South Kensington, London SW7 2AZ, UK \\ ${ }^{\mathrm{b}}$ Department of Mechanical Engineering, Imperial \\ College London, South Kensington, London SW7 2AZ, \\ UK \\ ${ }^{\mathrm{c}}$ Department of Electrical \& Electronic Engineering, \\ Imperial College London, South Kensington, London \\ SW7 2AZ, UK \\ ${ }^{\mathrm{d}}$ Department of Computing, Imperial College London, \\ South Kensington, London SW7 2AZ, UK, \\ ${ }^{\mathrm{e}}$ Wooden Spoon, Sentinel House, Ancels Business Park, \\ Harvest Crescent, Fleet, Hampshire, GU51 2UZ, UK
}

Background: The Assistive Technology (AT) market will surpass $\$ 26$ billion by 2024 (Forbes, March 2017), with over 1 billion people globally requiring assistive products. This sizeable market is wide ranging and disparate due to the diversity of the end user needs. Successful development and application of AT products for people with disabilities is made difficult by the complexities of the market.

The Internet of Things (IoT) equipment market is estimated to be $\$ 520$ billion by 2021 (Forbes, August 2018). The explosive growth of the IoT is due to its potential to connect things-to-things, people-to-things and people-to-people. However, few IoT developments take into consideration people with disabilities. There are IoT connectivity solutions available for some products but there is a lack of connection with AT Input Devices. Hence, the benefits of IoT are still yet to be leveraged to its maximum potential in promoting independent living.

There is a need for an open source software package capable of linking AT Input Devices to any connectable device using programmable settings to allow flexibil- ity for users. Such a system would enable the development of new AT devices and provide accessibility to IoT devices for people with disabilities.

Method: At the request of children's charity Wooden Spoon, a multidisciplinary team of undergraduate students at Imperial College London developed an open source software platform to allow the control of output devices via a variety of AT Input Devices in a plug-andplay fashion. The software is built as a web or desktop application so can be used on any computer or smartphone. Users can use their own specific input devices such as buddy buttons, puff and suck switches or eye trackers to control a variety output devices all via one application.

Key results: Three demonstration projects were developed using the system to show its potential:

1. Control of a Phillips smart lamp,

2. Interaction with Alexa using a Tobii eye tracker to type commands,

3. Control of a robotic arm using an AT joystick through the interface.

A demonstration was run for the staff at the Ace Centre, a charity specializing in AT, this provided positive and insightful feedback which highlighted the importance of accessibility of the system settings and enabling customization by the user to meet their needs. These concepts where then were integrated into the following iteration of the system. The feedback indicated that the system would have value to users, developers and suppliers.

Conclusion: A platform technology has been developed which enables the connection of conventional AT Input Devices, such as joysticks and buddy buttons, with IoT connected Output Devices, such as smart lamps and robotic arms. The aim is for this software to be released under an open source licence to allow users free access to it and to promote further development and connectivity within the AT field. A successful proof of concept demonstration was delivered in the first phase of this project with three examples of its ap- 
plication. The development is to continue in partnership with the Apperta Foundation.

Keywords: Control, Open Source, IoT, Connectivity. *Corresponding author. E-mail: i.radcliffe@imperial. ac.uk

\section{Implementing an IoT Based Task Analysis System to Promote Autonomy in Daily Hygiene of Adults with Autism in a Residential House \\ Federica Somma $^{\mathrm{a}, *}$, Angelo Rega ${ }^{\mathrm{a}}$, Onofrio Gigliotta ${ }^{\mathrm{a}}$ and Giuseppina Nappi ${ }^{\mathrm{b}}$ \\ ${ }^{a}$ Department of Humanistic Studies, University of Naples Federico II, Naples, Italy \\ ${ }^{\mathrm{b}}$ AIAS Onlus, Nola, Naples, Italy}

Background: The Internet of Things, IoT, has been increasingly gaining attention in educational contexts because it allows to connect physical objects through a network to enrich users' experience. Recently, particular attention has been placed to wearable objects, accessorizes provided with intelligent devices, such as beacons: usually bluetooth devices able to transmit and receive signals within short distances. IoT can improve the lives of people with disabilities. A good quality of life must include the possibility of being independent in all contexts and activities such as personal hygiene, dressing and undressing activities, moving in space. Unfortunately, autonomy is often lacking in people with autism.

To support the learning process of daily living activities of people with autism, it is important to facilitate a task, for example through a task analysis that analyzes and describes the sequence that composes an action, in the logical order for its correct execution. Our purpose is to verify whether wearable intelligent objects, complemented with a task analysis system, can be used, in a residential context, to facilitate everyday hygiene in individuals with severe autism.

Method: Participants will be 2 adults with severe autism. A single-subject study was chosen because it is more suitable for the development of technologies for severe disability. The experimentation will take place in the Residential Section of the A.I.A.S. Center (Nola, Naples), which offers a suitable housing solution. The essential tools for the study will be the beacons, produced by the Estimote company and already used in the center, and 1 10-inch tablet containing a software for task analysis. The task analysis will consist of a subdivision into small steps of daily hygiene tasks, such as brushing teeth. Whenever the participants will en- ter the bathroom, the tablet will retain the sensor signal and activate by playing on the tablet videos of all steps of the task. The participant will be able to observe the sequence and asked to reproduce it.

Key results: We expect that the beacon system, complemented with the task analyses software, will facilitate the daily hygiene skills of participants with severe autism, thus favoring their personal autonomy, and gradually helping them to generalize these skills in other contexts.

Conclusion: Internet of Things will impact on our everyday life. Those who already have some devices, connected to the Internet, already appreciate the possibility to remotely control their own home devices. However, we think that more advantage can be gained by people with special needs.

The purpose of the residential structure is to implement a global therapeutic, rehabilitative and educational life project. The use of the IoT system allows the person to take advantage of the help of the surrounding environment for accomplishing his specific task without relying on the human operator. The final goal of our work, however, is to promote independence also from our system, in fact, once individuals will succeed in their tasks the artificial support will be gradually removed to let them back to their usual contexts with new skills.

Keywords: IoT, Task Analysis, ASD, Beacon, Rehabilitation.

*Corresponding author. E-mail: federica.somma@ unina.it

The use of Internet of Things (IoT) and Assistive Technology (AT) in developing 'Smart Homes' for health and social care in the UK

George Nightingale ${ }^{\mathrm{a}, *}$, Tahira Resalat ${ }^{\mathrm{a}, *}$, Simon $\mathrm{Ng}^{\mathrm{a}, *}$, Dan Habbershaw ${ }^{\mathrm{a}, *}$, Germayne WilliamSylvester $^{\mathrm{a}, *}$ and Aejaz Zahid ${ }^{\mathrm{b}}$

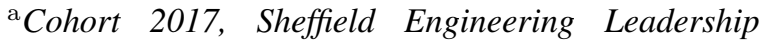
Academy, The University of Sheffield, Sheffield, UK

${ }^{\mathrm{b}}$ Centre for Assistive Technology and Connected Healthcare, The University of Sheffield, Sheffield, UK

Background: Consumer smart technology is a rapidly growing industry and becoming increasingly present in our day to day lives. The convenience this presents is hugely beneficial, but it can be utilised in a far more valuable way; to increase the independence of individuals with physical or cognitive disabilities in their homes, as well as monitor their lifestyle to assist in 
their care. However, using this technology in an assistive way to build an ecosystem of smart devices and sensors using Internet of Things (IoT) technology is an unfamiliar concept, unknown to both health and social care providers and the general public. The digital infrastructure required to realise this idea is also not currently available to the majority of regional care providers in the UK. This paper presents a case study where assistive technology and IoT are being used to address the challenges of providing health and social care in Barnsley Metropolitan Borough Council.

Method: The aim was to simulate a smart-home test bed that can demonstrate to care providers across the country, the capability of consumer IoT technologies and the benefit they provide to increasing the quality of care. Initially, interviews were conducted with members of Barnsley Council and health and social care professionals, as well as research to explore the multifaceted capabilities of consumer technologies to aid with lifestyle monitoring and residential assistance. Key target areas were identified based on the proportion of resources being spent on health and social care. Within this study, a scale physical model of a smart home was created, containing sensors to mimic current consumer technology. This model house served as a testbed that facilitated easy and quick simulation in a number of configurations. These sensors were evaluated on their ability to fulfil the monitoring needs of patients with frailty, dementia, learning disabilities, and mental health issues. A feasibility case was also built to help catalyse the process of implementing these new technologies amongst the services of healthcare providers across the country. Finally, an IoT digital framework was designed which would be accessible to prospective patients and social care bodies, working on a feedback loop system to provide information about the optimum technology available to suit each need.

Conclusion: The future of residential healthcare may lie within the optimisation of AT using IoT. In this study, we are developing this through linking a digital IoT framework with data collected from a simulated smart home environment. Although this study is only the first step towards a more efficient system, we believe that IoT may be integral to providing patients the independence they may not currently be receiving.

Keywords: IoT, residential-healthcare, smart-technology, lifestyle-monitoring, alzheimer's

*Corresponding author. E-mail: tahiraresalat@gmail. com
Waking-up in the Morning: A Gamified Simulation in the Context of Learning Activities of Daily Living

Polyxeni Kaimara ${ }^{\mathrm{a}, *}$, George Miliotis $^{\mathrm{b}}$, Ioannis Deliyannis ${ }^{\mathrm{c}}$, Emmanuel Fokides ${ }^{\mathrm{d}}$, Andreas C. Oikonomou $^{\mathrm{e}}$, Agnes Papadopoulou $^{\mathrm{f}}$ and Andreas Floros ${ }^{\mathrm{g}}$

a,b,c,f,g Department of Audiovisual Arts, Ionian University, Tsirigoti Sq. 7, 49100 Corfu, Greece

${ }^{\mathrm{d}}$ Department of Primary School Education, University of the Aegean, 1 Dimokratias str., 85132, Rhodes, Greece

${ }^{\mathrm{e}}$ Department of Education, School of Pedagogical and Technological Education (ASPETE), Papanastasiou 13, 54639 Thessaloniki, Greece

Background: The degree of child independence in Activities of Daily Living (ADLs) is crucial for parents, caregivers, educators, and therapists. Achieving basic skills is vital not only for children with developmental disabilities but also for typically developing preschoolers and primary school students. People with developmental disorders often have difficulties while performing ADLs, (e.g. dressing, cooking, cleaning and personal hygiene), whose acquisition leads to increased independence; therefore teaching focuses on conveying functional skills.

The relevant literature suggests that children with developmental disabilities face the following difficulties:

- comprehension of symbolic play and pretense

- low imagination level

- resistance to changes to their environment or daily routines

- limited ability to identify with others

- trouble applying what they have learned to real life

Three instructional approaches are commonly applied to promote daily living skills: (1) in-vivo instruction, (2) video-based instruction (VBI) and (3) computerbased intervention $(C B I)$. The problem with all three approaches individually is that application of taught skills to real life is very difficult for children with a limited ability to identify with others.

In order to overcome this difficulty, we are designing and developing an interactive simulation game called "Waking-up in the Morning" with image fidelity to ensure natural representation and better transfer of skills to real-world conditions. Our research questions are listed below:

- can the game function as educational material in the context of ADLs, both for students with special educational needs and their typically developing peers? 
- can gaming become the bridge between children regardless of their cognitive profile, promoting collaborative learning through peer-mentoring?

At this stage, alongside the game development per se, several of its aspects are examined (e.g., gamification techniques and interface design), taking into account users' evaluations and feedback (students with developmental disabilities and typically developing students) and game design experts.

Method: Our main goal is to investigate transmedia learning in inclusive conditions through gamified content that combines the three instructional approaches (in-vivo instruction, VBI and $C B I$ ), traditional gaming modes and cutting-edge technologies: cards, virtual/augmented reality and $360^{\circ}$ interactive videos. VR-enabled headsets, laptops, tablets, smartphones and interactive whiteboards will be used, depending on the player-learner's characteristics. Our literature review establishes the benefits that emerging technologies bring to individuals with special educational needs, as well as obstacles they face in the use of
VR devices, which are necessary to support novel gaming methodologies. The target group combines typically developing children aged seven years old and individuals with developmental disabilities of similar mental age. The simulation game will be tested in inclusive environments in general schools.

Conclusion: The purpose of this paper is to present our alternative approach, game design and production for teaching ADLs using cutting-edge technology. In the near future, we will examine if the proposed simulation game can be used to support different learning styles and differentiated instruction. The ultimate goal of the research is to suggest good practices in the field of gamified and highly interactive digital learning materials for the implementation of inclusive education.

Keywords: Activities of Daily Living, Interactive $360^{\circ}$ Game, Simulation, Transmedia Learning, Virtual/Augmented Reality.

*Corresponding author. E-mail: x_kaimara@yahoo. com 